\title{
A Comparative Effectiveness Study of Rescue Strategies in 1,000 Subjects With Severe Hypoxemic Respiratory Failure
}

\author{
Pablo Moreno Franco MD, Felicity Enders PhD, Gregory Wilson RRT, Ognjen Gajic MD, and \\ Sonal R Pannu MD
}

\begin{abstract}
BACKGROUND: Subjects with severe hypoxemic respiratory failure have shown a high mortality in previous studies. METHODS: All adult ICU patients requiring mechanical ventilation from 2005 to 2010 at Mayo Clinic were screened for severe hypoxemia (Murray lung injury score of $\geq 3$ ). Extracorporeal membrane oxygenation, prone positioning, high-frequency oscillatory ventilation (HFOV), and inhaled vasodilators were considered as rescue strategies. A propensity-based scoring was created for the indication or predilection to use each strategy. A model was created to evaluate the association of each rescue strategy with hospital mortality. RESULTS: Among 1,032 subjects with severe hypoxemia, 239 subjects received some form of rescue strategy (59 received a combination of therapies, and 180 received individual therapies). Inhaled vasodilators were the most common, followed by HFOV. Rescue strategies were used in younger subjects with severe oxygenation deficits. Subjects receiving rescue strategies had higher mortality and longer ICU stays. None of the strategies individually or in combination showed a significant association with hospital mortality after adjusting covariates by propensity scoring. Adjusted Odds ratios and respective 95\% CI were as follows: HFOV $0.67(0.35-1.27)$, extracorporeal membrane oxygenation $0.63(0.18-$ 1.92), prone position $1.07(0.49-2.28)$, and inhaled vasodilators $1.17(0.78-1.77)$. CONCLUSIONS: In this retrospective comparative effectiveness study, there was no association of rescue strategies with hospital mortality in subjects with severe hypoxemia. Key words: severe hypoxemic respiratory failure; rescue strategies; propensity modeling. [Respir Care 2016;61(2):127-133. () 2016 Daedalus Enterprises]
\end{abstract}

\section{Introduction}

Severe hypoxemic respiratory failure presents with the inability to achieve adequate arterial oxygenation in spite of high inspired oxygen or because of barotraumas. ${ }^{1}$ ARDS

Dr Moreno Franco is affiliated with the Departments of Transplant and Critical Care Medicine, Mayo Clinic, Jacksonville, Florida; Drs Moreno Franco, Gajic, and Pannu are affiliated with METRIC (Multidisciplinary Epidemiology and Translational Research in Intensive Care), Emergency and Perioperative Medicine, Mayo Clinic, Rochester, Minnesota; Dr Enders is affiliated with Biomedical Statistics and Informatics, Mayo Clinic, Rochester, Minnesota; Mr Wilson is affiliated with Respiratory Care, Mayo Clinic, Rochester, Minnesota; Drs Gacic and Pannu are affiliated with the Division of Pulmonary Care \& Critical Medicine, Mayo Clinic, Rochester, Minnesota; Dr Pannu is affiliated with the Division of Pulmonary Care \& Critical Medicine, Ohio State University, Wexner Medical Center, Columbus, Ohio.

Supplementary material related to this paper is available at http:// www.rcjournal.com

The authors have disclosed no conflicts of interest. is one of the major causes of severe hypoxemia and affects approximately 140,000 patients each year, with an overall mortality of approximately $40 \%$; $16 \%$ of these patients die as a result of severe hypoxemia. ${ }^{2}$ Previous studies using various rescue strategies have not shown a mortality benefit, with the exception of extracorporeal life support and prone position. ${ }^{3,4}$ A more recent study performed as part of the National Heart, Lung, and Blood Institute ARDS Clinical Trials Network (ARDSnet) showed that the patterns of rescue strategy utilization appear to be changing over time, but failed to find evidence of survival benefit in acute lung injury. ${ }^{5}$ In this study, our aim was to investigate utilization patterns and outcomes associated with rescue strategies in severe hypoxemia.

Correspondence: Pablo Moreno Franco MD, Transplant \& Critical Care Medicine, Mayo Clinic, 4500 San Pablo Road, Jacksonville, FL 32224. E-mail: morenofranco.pablo@mayo.edu.

DOI: $10.4187 /$ respcare. 04162 


\section{Methods}

\section{Study Design}

In this retrospective comparative effectiveness study, consecutive subjects with severe hypoxemia were included. Data variables were extracted from the institutional electronic medical record database, the METRIC Data Mart, ${ }^{6}$ as well as individual case review. All work was performed at Mayo Clinic (Rochester, Minnesota). This study was approved by Mayo Clinic's institutional review board (10-006712). (See the supplementary materials at http:// www.rcjournal.com for additional details.)

\section{See the Related Editorial on Page 255}

\section{Patient Selection}

All adult ICU patients at Mayo Clinic requiring mechanical ventilation from 2005 to 2010 were identified by the Mayo Clinic ICU Data Mart. ${ }^{6}$ All patients younger than $18 \mathrm{y}$ of age and those who refused the use of their medical records for research were excluded. An independent observer verified the validity of all subject data. Only the first ICU admission for each subject within these $6 \mathrm{y}$ was included. Any readmission within $24 \mathrm{~h}$ was considered part of the initial ICU course. (See the supplementary materials at http://www.rcjournal.com for details on patient selection.)

\section{Definition of Severe Hypoxemia and ARDS}

Severe hypoxemia was defined as the Murray Lung Injury Score (LIS) of 3 or more on at least one occasion during the subject's ICU stay.,7 The process for identifying subjects with refractory hypoxemia is outlined in Figure 1. After identification of these candidate subjects, a 4-component LIS was calculated and subjects with an LIS $\geq 3$ were defined as having severe hypoxemia. The presence of ARDS was confirmed when subjects met the 1994 American-European Consensus Conference criteria. ${ }^{3,4,6-9}$ Subjects were determined to have possible cardiogenic pulmonary edema if they had significant mitral or aortic valve dysfunction or left ventricular heart failure on an echocardiogram obtained within 2 weeks of the ICU stay. Echocardiographic images were reviewed by a research cardiologist specialized in echocardiography. Systolic left ventricular heart failure was defined as a left ventricular ejection of $<40 \%$. Diastolic left ventricular heart failure was defined as an e/e' ratio of 15 or higher. In subjects without an echocardiogram, ARDS as the predominant cause of respiratory failure was established if the presence of known ARDS risk factor was noted.

\section{QUICK LOOK}

\section{Current knowledge}

Severe acute respiratory distress syndrome with refractory hypoxemia is characterized by a $\mathrm{P}_{\mathrm{aO}_{2}} / \mathrm{F}_{\mathrm{IO}_{2}}<100$ despite optimization of PEEP and $\mathrm{F}_{\mathrm{IO}_{2}}$. Common rescue strategies for refractory hypoxemia include prone positioning, high-frequency oscillatory ventilation, airway pressure release ventilation, inhaled vasodilators, and extracorporeal membrane oxygenation.

\section{What this paper contributes to our knowledge}

In a group of $>1,000$ subjects with refractory hypoxemia, one or more rescue strategies were used in $23 \%$ of subjects. Inhaled vasodilators were the most common rescue strategy and were frequently combined with either highfrequency oscillatory ventilation or prone positioning. No survival benefit was found for the use of rescue strategies.

\section{Measurements}

In-hospital mortality was the main outcome variable. Rescue strategies were defined as use of any: high-frequency oscillatory ventilation (HFOV), extracorporeal membrane oxygenation (ECMO), prone positioning, or inhaled vasodilators (nitric oxide and/or prostacyclin).

Each rescue strategy was searched electronically via their respective databases. HFOV and ECMO were searched through the METRIC Data Mart, and ECMO was also searched through the Mayo Clinic ECMO records. Prone positioning was searched using electronic data retrieval through the Mayo Clinic Life Sciences System, a sophisticated data warehouse. This approach has been previously validated. ${ }^{6}$ The electronic data retrieval was used to form a query for the terms "prone position" and "rota prone." The Mayo Clinic Respiratory Care database was used to search for use of inhaled nitric oxide and inhaled prostacyclin. Electronic charts of subjects receiving rescue strategies were verified manually by independent reviewers.

\section{Data Collection}

Demographic data and cardiovascular, hemodynamic, and pulmonary variables were recorded from the METRIC Data Mart. Continuous values were extracted, and then a 24-h median was calculated. Manual validation was conducted for 5 different percent values at each extreme of the range. Standardized definitions and previously validated web-based automated search strategies utilizing electronic data retrieval were used for collecting comorbid risk factors and related diagnoses. ${ }^{10}$ We used the estimated deadspace ventilation as validated by Siddiki et al. ${ }^{11}$ 


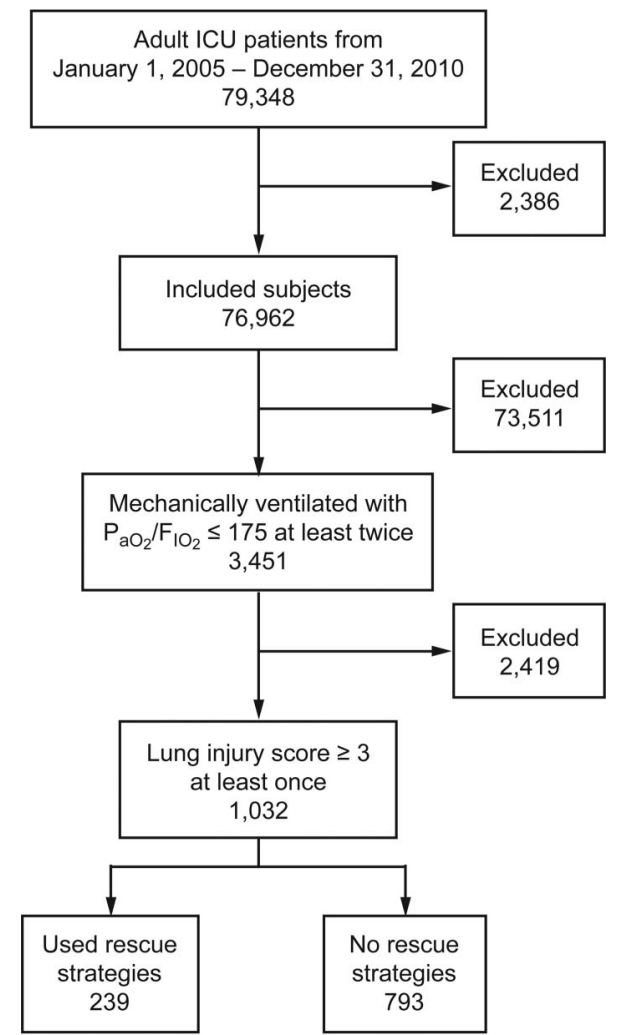

Fig. 1. Flow chart. Figure shows use of rescue therapies in refractory hypoxemia at Mayo Clinic (2005-2010).

\section{Statistical Analysis}

Continuous and categorical variables were compared using the Wilcoxon, chi-square, or Fisher exact test, as appropriate, between subjects with and without rescue strategies. A propensity score was made for the use of each therapy as mentioned in the following section. We conducted univariate and multivariate analyses using the propensity score.

\section{Propensity Score}

Covariate adjustment using the propensity score was done to adjust for probability of receiving individual rescue strategies. The covariate for creating the propensity score for each therapy was based on clinical relevance, physiological basis, and known practice patterns. The propensity score for each rescue strategy consists of the particular rescue strategy as the outcome variable and the clinical factors leading to its use as the predictor variables. A modified version of the Severe Hypoxemia Related Risk Prediction (SHARP) model, consisting of pulmonary dead-space fraction, oxygenation index, arterial $\mathrm{pH}$, and vasopressor use, was used to adjust for severity of illness in all the propensity scores.

The propensity models were made based on the following criteria: a history of congestive heart failure or the presence of heart failure on the particular hospital admission were covariates for ECMO; the presence of pulmonary hypertension was a covariate for the use of inhaled vasodilators; because prone positioning is a predominant practice at the general surgical ICU and trauma ICU at Mayo Clinic, admission to the general surgical ICU or trauma ICU was considered a covariate for prone positioning; utilization of high PEEP and admission to the medical or mixed medical ICU were covariates for HFOV. The probability for use of each therapy, derived from the individual models, was then plotted against the use of each therapy. Each of the propensity scores, along with use of each therapy and acute physiology and chronic health evaluation (APACHE) III, were then included in the final model as predictor variables with hospital mortality as the outcome variable.

\section{Results}

In this comparative effectiveness trial, we screened 79,846 adult ICU patients from January 2005 to December 2010. A total of 1,032 subjects had an LIS score of 3 or higher. ARDS was the cause of severe hypoxemia in 793 subjects. Of the 1,032 subjects, $239(\sim 23 \%)$ received at least one of the four categories of rescue strategy (Fig. 1). Among the 239 subjects, 59 received a combination of therapies and 180 received individual therapies (Fig. 2A). Inhaled vasodilators were the most common category of rescue strategies given in 189 subjects. HFOV was the next most used in 61 subjects. Prone position and ECMO were used in 42 and 16 subjects, respectively (Fig. 2B). No specific patterns were noted when utilization was trended from 2005 to 2010 (Fig. 3).

Demographics and baseline characteristics of subjects who received rescue strategies are noted in Table 1. The median age of rescue strategies subjects was 56 y (IQR 45-67), which is lower than the median age of subjects who did not receive rescue strategies (62 y, IQR 50-74). Both groups were similar in distribution of gender, body mass index, and severity of illness, as noted by their APACHE III and sequential organ failure assessment scores on day 1. Subjects in whom rescue strategies were used had a higher incidence of pulmonary hypertension as a co-morbid condition on ICU admission. Those with rescue strategies had a lower incidence of aspiration as a cause of respiratory failure, but had a higher incidence of sepsis and shock. All subjects received sedative agents, and nearly all received paralytics. However, this was not standardized or in the form of a protocol and was not documented in a way that could be easily collected for post-hoc analysis.

Rescue strategies subjects had fewer ventilator-free days and longer ICU stays; however, the hospital stay was similar in both groups. Although both groups had comparable tidal volume use and PEEP, the group with rescue strate- 

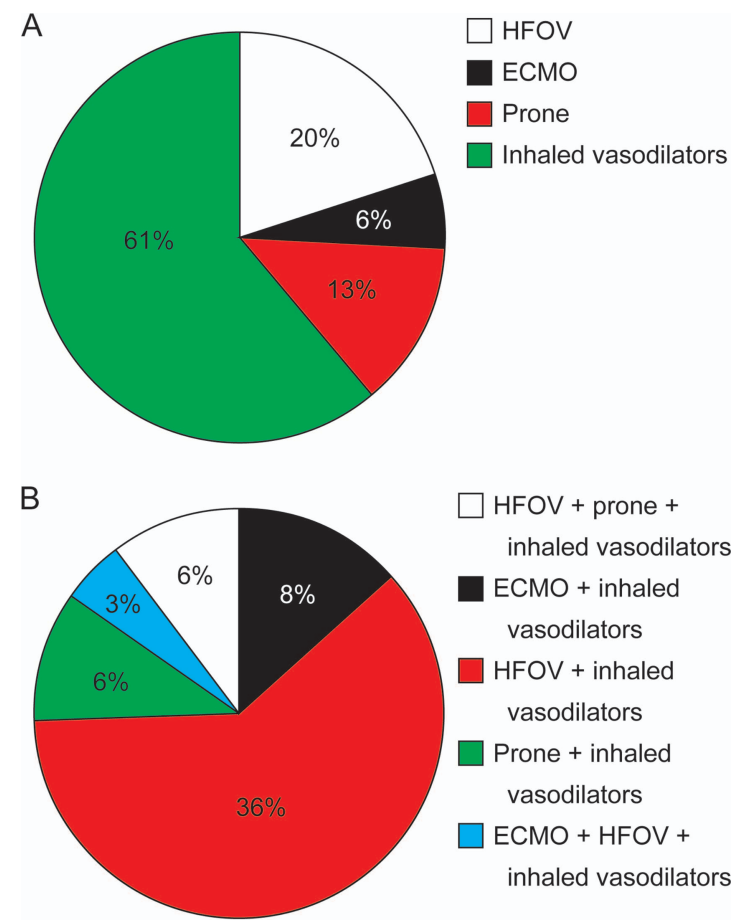

Fig. 2. Rescue strategies for severe hypoxemic respiratory failure at Mayo Clinic (2005-2010). (A) Distribution of 308 rescue strategy events. (B) Distribution of combinations of rescue strategies. $\mathrm{HFOV}=$ high-frequency oscillatory ventilation; $\mathrm{ECMO}=$ extracorporeal membrane oxygenation.

gies had higher plateau and peak pressures. They were more hypoxemic and had higher dead-space fractions. Mortality was higher in the rescue strategies group (Table 1).

The use of inhaled vasodilators, prone positioning, and HFOV correlated with increasing propensity; however, the same was not noted for ECMO, possibly because there were fewer subjects on ECMO. On creation of the propensity score, none of the therapies individually or in combination showed a significant association with adjusted hospital mortality. This remained true even when ECMO was removed from the model (Table 2).

\section{Discussion}

In our study, rescue strategy use was noted in approximately $23 \%$ of subjects with severe hypoxemia, and remained unchanged when restricted to ARDS only. This number is higher than that noted in the ARDSnet studies $(6.3 \%) .{ }^{5}$ Three significant differences leading to this finding are: (1) our subjects had more profound and persistent hypoxemia; (2) previously published data were a secondary analysis of multi-center, randomized, controlled trial data from ARDSnet studies, whereas ours was an observational study; and (3) the proportion of subjects who received combination therapy was higher in our group $(25 \%$ vs $15 \%){ }^{12}$
Inhaled vasodilators were noted to be the most commonly used modality overall. In addition to addressing pulmonary hypertension associated with ARDS or severe hypoxemia, human factors such as ease of implementation, expertise of respiratory therapists, and ability to combine with other strategies, such as HFOV, prone, or ECMO, have presumably played a role in this finding. Our results are different from those reported from ARDSnet trials, where the most frequently used rescue strategy was prone positioning in $58 \%$ of subjects. ${ }^{5}$

HFOV was the second most commonly used, predominantly in the medical ICU and mixed medical ICU (hematology/oncology)..$^{12}$ This could reflect the practice preferences of a closed group of intensivists working at both locations. This practice trend was prior to the large multicenter randomized trial in early ARDS that was terminated prematurely due to harm. ${ }^{3}$ The use of HFOV was much more frequent in our population than in the ARDSnet study ( $25 \%$ vs $7.2 \%)$.

Prone position was noted at large in the trauma and general surgical ICU. This again reflects the practice preferences of a closed group of intensivists. When compared with the study by Walkey et $\mathrm{al},{ }^{5}$ in our population, the use of prone position was much less frequent (58\% vs 16\%). In our cohort, the use of ECMO remained comparable with previously analyzed data from clinical trials. ${ }^{5}$ These data, again, predate the efficacy and economic assessment of conventional ventilatory support versus ECMO for severe adult respiratory failure (CESAR) study for ECMO in ARDS. ${ }^{3}$ This prevalence of ECMO use is likely representative of the complex nature of the intervention, second or third tier approach toward ECMO as a rescue strategy, as well as restricted inclusion criteria for EMCO.

Over the 6-y period, we noted overall high use of inhaled vasodilators and low use of ECMO. No significant upward or downward trends were noted. This is different from trends noted in the ARDSnet trial of increasing prone positioning use and decreasing inhaled vasodilators from 1996 to $2005 .^{5}$ Our results were similar to the ones noted in Europe during a similar time period, where prone position use did not change significantly. ${ }^{13}$

We noted that, generally, rescue strategies were used in younger subjects (adjusted odds ratio 0.81 [0.74-0.89]) with higher oxygenation deficit as noted previously. ${ }^{5}$ Those with rescue strategies had a higher incidence of pulmonary hypertension as a co-morbid condition on admission. Thus, it is likely that the pulmonary hypertension has contributed to the hypoxemia and thus higher use of rescue strategies. We are not clearly able to delineate the cause of pulmonary hypertension from our data retrieval. There was lower incidence of aspiration as an etiology for respiratory failure in subjects in whom rescue strategies were used. Hypoxemia when caused by aspiration has been noted to be of lesser severity, and therefore likely led to the lesser 


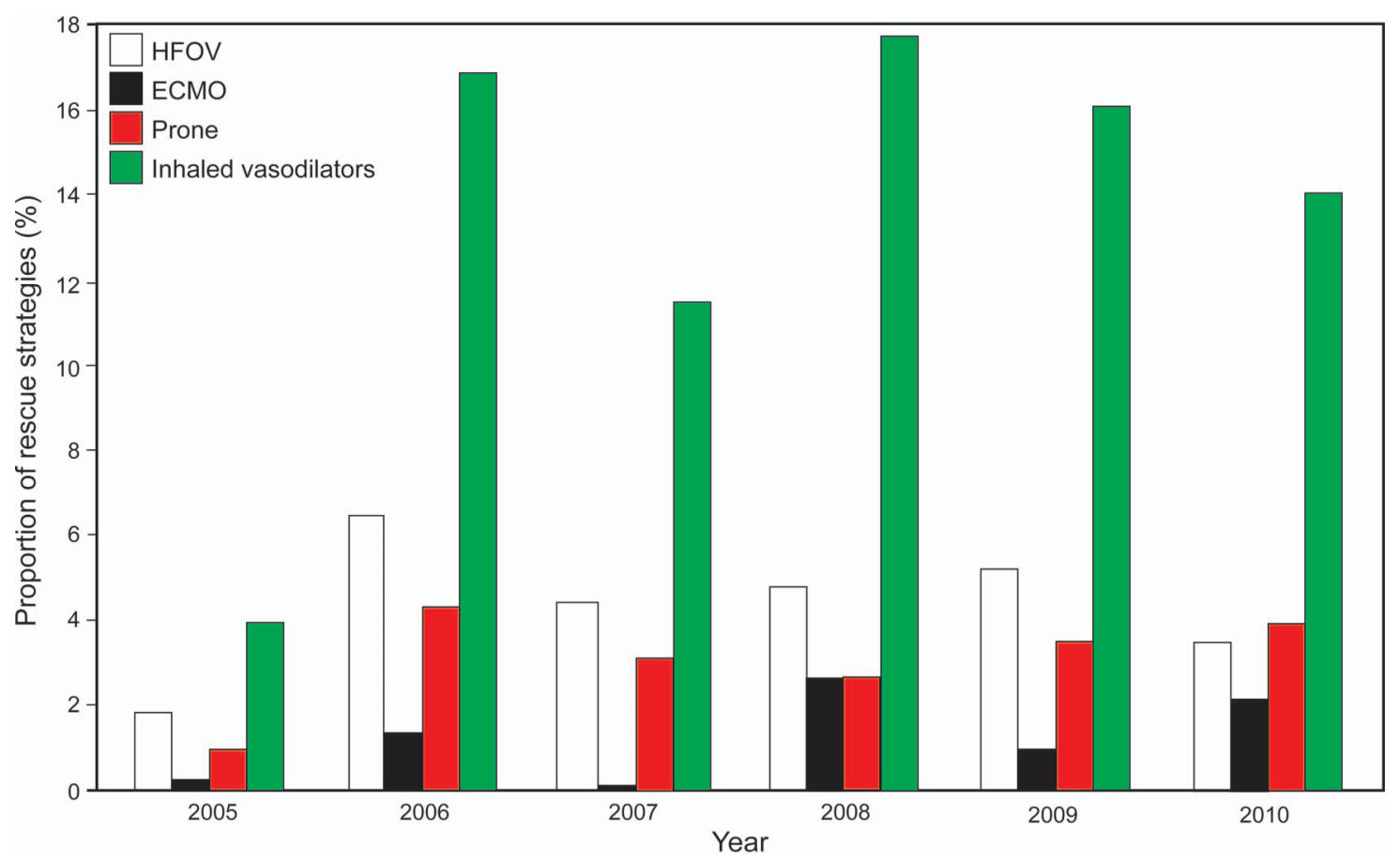

Fig. 3. Trends of rescue strategies at Mayo Clinic from 2005 to 2010 . HFOV = high-frequency oscillatory ventilation; ECMO $=$ extracorporeal membrane oxygenation.

requirement of rescue strategies. ${ }^{14}$ The incidence of COPD and interstitial lung disease (ILD) in our population is relatively higher. These subjects may have pulmonary hypertension as a contributing factor. Although none of these subjects were admitted for COPD exacerbation, the presence of exacerbation in either of these conditions cannot be excluded in acute respiratory failure. Both COPD and ILD provide relative contraindications to rescue strategies involving high PEEP and high mean airway pressure, with concerns of barotrauma. Involvement of these patients in trials of rescue strategies should be considered, but with caution.

No single rescue strategy showed significant association with reduced hospital/ICU mortality. The mortality in our study among subjects with rescue strategies was $47.7 \%$, comparable with previous literature and higher than that in the group of subjects without rescue strategy use. ${ }^{15} \mathrm{We}$ contemplate that, as compared with subjects without rescue strategies, subjects with rescue strategies had more profound oxygenation deficits and higher severity of illness scores, causing residual confounding despite statistical adjustment. It is certainly possible that worse outcomes can also be the result of adverse effects of rescue strategy; however, this cannot be proven based on our data. ${ }^{2}$ In ARDS, previous HFOV trials had equivocal data on mortality, but the most recent study was terminated due to harm. ${ }^{16,17}$ Similarly, inhaled vasodilators have never been associated with improved survival. ${ }^{18}$ ECMO use in the CESAR, in experienced centers, did show improved sur- vival, but the study has been condemned for absence of standardized management in the control arm. ${ }^{3}$ Prone positioning had not previously shown mortality benefit; however, when restricted to severely hypoxemic subjects $\left(\mathrm{P}_{\mathrm{aO}_{2}} / \mathrm{F}_{\mathrm{IO}_{2}}<100\right)$ and when given for at least $16 \mathrm{~h}$ in subjects with a $\mathrm{P}_{\mathrm{aO}} / \mathrm{F}_{\mathrm{IO}_{2}}<150$, there were improved outcomes. ${ }^{4,19}$

Our study has some potential limitations. Unfortunately, we do not have data on therapy limitations for all 1,032 subjects. Next, the presence of human immunodeficiency virus and acquired immunodeficiency syndrome in our population is extremely low $(<5 \%)$, so specific pathophysiology in respiratory failure associated with them may not be represented. However, our cohort does contain subjects with single or multiple organ transplantation, with a comparable degree of immunosuppression or opportunistic infections. Furthermore, the studied patient population was mostly white (approximately 80\%). There can be inherent constraints associated with data mining and electronic web-based queries. However, the queries we used have been validated and shown to be highly accurate and efficient for data collection. ${ }^{11}$ We tried to reduce the possibility of any incorrect data extraction by using a previously validated database (METRIC Data Mart) and randomly manually verifying $5 \%$ of the data. ${ }^{3}$ All the subjects with rescue strategies were also manually verified. Also based on the findings of this study, the reason for preference of specific rescue strategies cannot be deter- 
Table 1. Baseline Characteristics, Risk Factors, and Outcomes in Subjects With and Without Rescue Strategies

\begin{tabular}{|c|c|c|c|}
\hline Subject Characteristics & $\begin{array}{l}\text { Rescue Strategy } \\
\quad(n=239)\end{array}$ & $\begin{array}{l}\text { No Rescue Strategy } \\
\quad(n=793)\end{array}$ & $P$ \\
\hline Median age (IQR) & $56(45-67)$ & $62(50-74)$ & $<.001^{*}$ \\
\hline Gender, female $(\%)$ & $87(36.4)$ & $318(40.1)$ & .30 \\
\hline Median BMI (IQR) & $29.0(24.6-34.6)$ & $28.5(24.2-33.5)$ & .08 \\
\hline Median SOFA day 1 & $10(7-13)$ & $9(6-12)$ & .05 \\
\hline Median 24-h APACHE III & $96(75-119)$ & $94(75-115)$ & .65 \\
\hline \multicolumn{4}{|l|}{ Comorbid conditions, $n(\%)$} \\
\hline Pulmonary hypertension & $96(40.1 \%)$ & $197(24.8 \%)$ & $<.01 *$ \\
\hline Immunosuppression & $102(42.6)$ & $326(39.8)$ & .43 \\
\hline Lymphoma & $18(7.5)$ & $58(7.1)$ & .91 \\
\hline Leukemia & $20(8.3)$ & $61(7.6)$ & .73 \\
\hline Cirrhosis & $17(7.1)$ & $86(10.8)$ & .08 \\
\hline COPD & $62(25.9)$ & $212(26.7)$ & .72 \\
\hline DM & $105(23)$ & $169(30)$ & .80 \\
\hline ILD & $24(10.4)$ & $61(7.7)$ & .25 \\
\hline \multicolumn{4}{|l|}{ Conditions during ICU stay, $\mathrm{n}(\%)$} \\
\hline Sepsis & $142(59.4)$ & $408(51.4)$ & $.03 *$ \\
\hline Shock & $46(19.2)$ & $108(13.6)$ & $.03 *$ \\
\hline Pneumonia & $201(84.1)$ & $673(84.6)$ & .77 \\
\hline Pancreatitis & $37(15.4)$ & $110(13.8)$ & .53 \\
\hline Aspiration & $146(61.0)$ & $543(68.4)$ & $.03 *$ \\
\hline \multicolumn{4}{|l|}{ Mechanical ventilation-related variables } \\
\hline Median $\mathrm{V}_{\mathrm{T}} / \mathrm{PBW}(\mathrm{IQR})$ & $6.5(6.0-7.3)$ & $6.7(6.1-7.4)$ & .06 \\
\hline Median PEEP (IQR) & $12(8-15.5)$ & $10(7-10)$ & .09 \\
\hline Median plateau pressure (IQR) & $26(22-31)$ & $22(19-25)$ & $<.001$ \\
\hline Median $\mathrm{P}_{\mathrm{aO}_{2}} / \mathrm{F}_{\mathrm{IO}_{2}}(\mathrm{IQR})$ & $100(76-134)$ & $127(93-165)$ & $<.001$ \\
\hline Median OI (IQR) & $20.6(15-29.1)$ & $14.6(9.5-19)$ & $<.001^{*}$ \\
\hline Median estimated $\mathrm{V}_{\mathrm{D}} / \mathrm{V}_{\mathrm{T}}(\mathrm{IQR})$ & $0.63(0.54-0.72)$ & $0.58(0.46-0.67)$ & $<.001 *$ \\
\hline \multicolumn{4}{|l|}{ Outcomes } \\
\hline Hospital death $(\%)$ & $114(47.8)$ & $276(34.8)$ & $<.001 *$ \\
\hline Refractory hypoxemia hours before death (IQR) & $94.7(37.2-222.4)$ & $45.8(16.1-161.0)$ & $<.001^{*}$ \\
\hline Ventilator-free days (IQR) & $0(0-17.8)$ & $15.8(0-22.6)$ & $<.001 *$ \\
\hline Hospital LOS (IQR) & $20.1(9.46-37.6)$ & $17.5(9.09-31.1)$ & .06 \\
\hline ICU LOS (IQR) & $11.3(5.74-20.9)$ & $9.15(4.9-25.1)$ & $>.001 *$ \\
\hline $\begin{array}{l}\text { * Statistically significant. } \\
\text { IQR = interquartile range } \\
\text { BMI = body mass index } \\
\text { SOFA = Sequential Organ Failure Assessment } \\
\text { APACHE = Acute Physiology and Chronic Health Evaluation } \\
\text { DM = diabetes mellitus } \\
\text { ILD }=\text { interstitial lung disease } \\
\mathrm{V}_{\mathrm{T}} / \mathrm{PBW}=\text { tidal volume for predicted body weight } \\
\mathrm{OI}=\text { oxygenation index } \\
\mathrm{V}_{\mathrm{D}} / \mathrm{V}_{\mathrm{T}}=\text { estimated dead-space ventilation } \\
\mathrm{LOS}=\text { length of stay }\end{array}$ & & & \\
\hline
\end{tabular}

mined. The practice pattern could reflect the preferences of a closed group of intensivists.

To our knowledge, this is the first and largest study of subjects with severe hypoxemia of all causes evaluating comparative effectiveness of rescue strategies. It is also the first study to utilize a propensity modeling for the indication of rescue strategies in this population. This is particularly important in this setting, where indications for utilizing one therapy over another were largely determined by the physician caring for the patient. Some papers have tried to elucidate an algorithmic approach to managing severe hypoxemia, ${ }^{19}$ but it is unclear what the acceptance rate of such an approach is in actual clinical practice. Additionally, the study being performed at a tertiary medical center with no limitation in the availability of equipment and expertise may help reduce the bias of preference of a specific rescue strategy due to limited resources. 


\section{Rescue Strategies for Refractory Hypoxemia}

Table 2. Association of Rescue Strategies With Hospital Mortality

\begin{tabular}{lcc}
\hline \hline \multicolumn{1}{c}{ Rescue Strategy } & $\begin{array}{c}\text { Odds Ratio, 95\% CI } \\
\text { (Unadjusted) }\end{array}$ & $\begin{array}{c}\text { Odds Ratio, 95\% CI } \\
\text { (Adjusted)* }\end{array}$ \\
\hline HFOV $\dagger$ & $0.78(0.43-1.39)$ & $0.67(0.35-1.27)$ \\
ECMO $\$$ & $0.7(0.22-1.95)$ & $0.63(0.18-1.92)$ \\
Prone position§ & $1.19(0.59-2.35)$ & $1.07(0.49-2.28)$ \\
Inhaled nitric oxide and & $1.16(0.80-1.66)$ & $1.17(0.78-1.77)$ \\
$\quad$ prostaglandins $\|$ & &
\end{tabular}

\footnotetext{
* SHARP (Severe Hypoxemia Related Risk Prediction) model was used as a covariate in creating individual propensity scores for each rescue strategy.

APACHE (Acute Physiology and Chronic Health Evaluation) III and propensity score for each rescue therapy was added to the model.

$\dagger$ HFOV propensity $=$ use of high PEEP and admission to medical/mixed medical ICU.

$\ddagger \mathrm{ECMO}$ propensity $=$ presence of heart failure or history of congestive heart failure

$\S$ Prone propensity $=$ admission to the trauma/general surgery ICU.

|| Inhaled vasodilator propensity $=$ presence of pulmonary hypertension

$\mathrm{HFOV}=$ high-frequency oscillatory ventilation

$\mathrm{ECMO}=$ extracorporeal membrane oxygenation
}

\section{Conclusions}

In summary, rescue strategies continue to be used in a minority of subjects with severe hypoxemia, and, in general, there is no benefit to survival seen. However, there are a few studies out now that have used individual rescue strategies that are showing improved mortality in selected subsets of subjects. We believe further research toward an algorithmic and pathophysiology-based approach for the selection of rescue strategies in patients with severe hypoxemia is warranted.

\section{REFERENCES}

1. Esan A, Hess DR, Raoof S, George L, Sessler CN. Severe hypoxemic respiratory failure: part 1 -ventilatory strategies. Chest 2010; 137(5):1203-1216.

2. Luhr OR, Antonsen K, Karlsson M, Aardal S, Thorsteinsson A, Frostell CG, Bonde J. Incidence and mortality after acute respiratory failure and acute respiratory distress syndrome in Sweden, Denmark, and Iceland; The ARF Study Group. Am J Respir Crit Care Med 1999;159(6):1849-1861.

3. Peek GJ, Mugford M, Tiruvoipati R, Wilson A, Allen E, Thalanany MM, et al. Efficacy and economic assessment of conventional ventilatory support versus extracorporeal membrane oxygenation for severe adult respiratory failure (CESAR): a multicentre randomised controlled trial. Lancet 2009;374(9698):1351-1363.

4. Guérin C, Reignier J, Richard JC, Beuret P, Gacouin A, Boulain T, et al. Prone positioning in severe acute respiratory distress syndrome. N Engl J Med 2013;368(23):2159-2168.
5. Walkey AJ, Wiener RS. Utilization patterns and patient outcomes associated with use of rescue therapies in acute lung injury. Crit Care Med 2011;39(6):1322-1328.

6. Herasevich V, Pickering BW, Dong Y, Peters SG, Gajic O. Informatics infrastructure for syndrome surveillance, decision support, reporting, and modeling of critical illness. Mayo Clin Proc 2010; 85(3):247-254.

7. Murray JF, Matthay MA, Luce JM, Flick MR. An expanded definition of the adult respiratory distress syndrome. Am Rev Respir Dis 1988;138(3):720-723.

8. Ferguson ND, Kacmarek RM, Chiche JD, Singh JM, Hallett DC, Mehta S, Stewart TE. Screening of ARDS patients using standardized ventilator settings: influence on enrollment in a clinical trial. Intensive Care Med 2004;30(6):1111-1116.

9. Trillo-Alvarez C, Cartin-Ceba R, Kor DJ, Kojicic M, Kashyap R, Thakur $\mathrm{S}$, et al. Acute lung injury prediction score: derivation and validation in a population-based sample. Eur Respir J 2011;37(3): 604-609.

10. Alsara A, Warner DO, Li G, Herasevich V, Gajic O, Kor DJ. Derivation and validation of automated electronic search strategies to identify pertinent risk factors for postoperative acute lung injury. Mayo Clin Proc 2011;86(5):382-388.

11. Siddiki H, Kojicic M, Li G, Yilmaz M, Thompson TB, Hubmayr $\mathrm{RD}$, et al. Bedside quantification of dead-space fraction using routine clinical data in patients with acute lung injury: secondary analysis of two prospective trials. Crit Care 2010;14(4):R141.

12. Raoof S, Goulet K, Esan A, Hess DR, Sessler CN. Severe hypoxemic respiratory failure: part 2-nonventilatory strategies. Chest 2010; 137(6):1437-1448.

13. Esteban A, Ferguson ND, Meade MO, Frutos-Vivar F, Apezteguia $\mathrm{C}$, Brochard L, et al. Evolution of mechanical ventilation in response to clinical research. Am J Respir Crit Care Med 2008;177(2):170177

14. Pannu SR, Moreno Franco P, Li G, Malinchoc M, Wilson G, Gajic $\mathrm{O}$. Development and validation of severe hypoxemia associated risk prediction model in 1,000 mechanically ventilated patients. Crit Care Med 2015;43(2):308-317.

15. Meade MO, Cook DJ, Guyatt GH, Slutsky AS, Arabi YM, Cooper DJ, et al. Ventilation strategy using low tidal volumes, recruitment maneuvers, and high positive end-expiratory pressure for acute lung injury and acute respiratory distress syndrome: a randomized controlled trial. JAMA 2008;299(6):637-645.

16. HiFO Study Group. Randomized study of high-frequency oscillatory ventilation in infants with severe respiratory distress syndrome. J Pediatr 1993;122(4):609-619.

17. Ferguson ND, Cook DJ, Guyatt GH, Mehta S, Hand L, Austin P, et al. High-frequency oscillation in early acute respiratory distress syndrome. N Engl J Med 2013;368(9):795-805.

18. Adhikari NK, Burns KE, Friedrich JO, Granton JT, Cook DJ, Meade MO. Effect of nitric oxide on oxygenation and mortality in acute lung injury: systematic review and meta-analysis. Br Med J 2007; 334(7597):779.

19. Gattinoni L, Tognoni G, Pesenti A, Taccone P, Mascheroni D, Labarta V, et al. Effect of prone positioning on the survival of patients with acute respiratory failure. N Engl J Med 2001;345(8):568-573.

This article is approved for Continuing Respiratory Care Education credit. For information and to obtain your CRCE

(free to AARC members) visit www.rcjournal.com

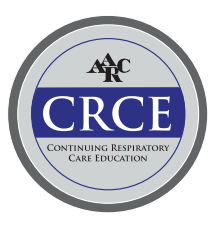

